\title{
INVESTIGATION OF DETECTION POSSIBILITY OF UAVS USING LOW COST MARINE RADAR
}

\author{
Andrius LAUČYS ${ }^{1 *}$, Saulius RUDYS ${ }^{1}$, Martynas KINKA ${ }^{1}$, Paulius RAGULIS ${ }^{1}$, \\ Jurgis ALEKSANDRAVIČIUSS ${ }^{1}$, Džiugas JABLONSKAS ${ }^{1}$, Domantas BRUČAS ${ }^{2}$, \\ Einius DAUGÉLA ${ }^{2}$, Laurynas MAČIULIS ${ }^{2}$ \\ ${ }^{1}$ Faculty of Physics, Vilnius University, Vilnius, Lithuania \\ ${ }^{2}$ Lithuanian Space Science and Technology Institute, Vilnius, Lithuania
}

Received 23 November 2018; accepted 13 May 2019

\begin{abstract}
The technologies of Unmanned Aerial Vehicles (UAVs) are fast emerging, but as any other technology, development of UAVs provides not only benefits but also the threats. UAV technologies are developing much faster than means of their control and detection. RADAR technology is one of the means of UAV's detection. Usually, radars are expensive, and usage of high-power radiation is problematic in many cases.

Today's market provides low cost marine radar working on various principles of operation. Such radar are not optimal, but could be used for UAV detection. Detection possibility of UAVs by FMCW marine radar was investigated by using two types of small UAVs as targets.
\end{abstract}

Keywords: UAV, FMCW, radar, marine radar, radar cross-section, UAV's detection.

\section{Introduction}

The technologies of Unmanned Aerial Vehicles (UAVs) saw extremely rapid development in recent years. The field of implementation of UAVs is especially wide, from "consumer drones" to extremely complex scientific or military applications. Unfortunately, same as any other technology, development of UAVs provides not only benefits but also the threats - smallest of which are the privacy invasion or flights above crowded areas. Much more damage can be done by UAV in the area of airport or any other critical infrastructure, carrying illegal items (like drugs) over the border or implemented as a tool by terrorists. UAV technologies are developing much faster than means of their control. For example electronic UAV control system U-SPACE is only at the concept at the moment. There are many means of UAV detection and neutralization suggested in the world, nonetheless all of them have some serious disadvantages. The essential problems in detection of UAVs - small size of UAVs, weak radio wave reflection (due to composite materials implemented), weak radio signal and sound emission.

\section{Means of detection}

Optic, acoustic, passive RF and radar detections are conventional means in UAV's detection technologies. Advantages and disadvantages of these technologies for micro UAVs detection are briefly presented below.

\subsection{Optic detection}

Optic detection can be performed from far infrared (thermal) to visible light spectrum. Usually, the optic detection systems are implemented as gimbal with a set of various spectral and view range cameras. Optical detection of the drones normally allows detecting them at the range up to $1000 \mathrm{~m}$, nevertheless it is highly dependable on the size of a UAV and (especially) weather conditions. Though UAVs are possible to detect using optical means at clear weather conditions (Figure 1), optical detection becomes practically impossible even at short distance in case of fog or cloud cover (even behind the vehicle) (Figure 2).

*Corresponding author. E-mail: andriuslaucys@gmail.com 


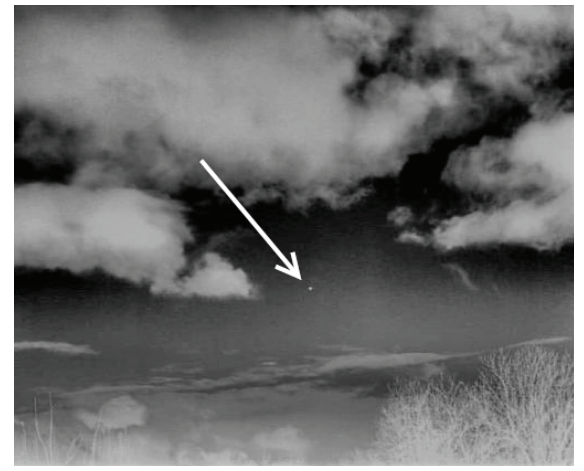

Figure 1. UAV at $200 \mathrm{~m}$. detected by LWIR (long wave infra-red, $20 \mathrm{~mm}$ focal distance) camera at clear sky behind DJI Phantom 3

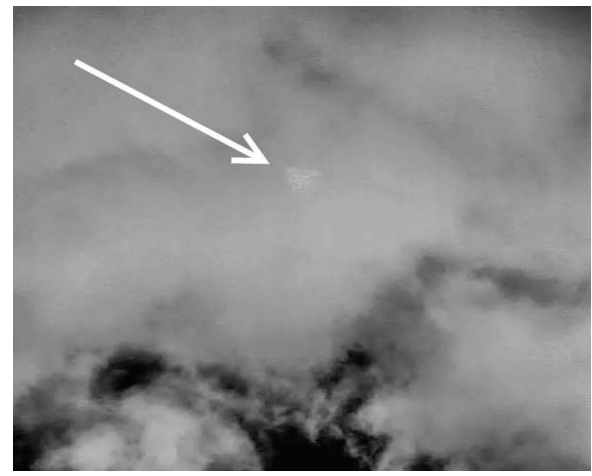

Figure 2. UAV barely detectable with the clouds at the back at $50 \mathrm{~m}$ distance (LWIR camera, $20 \mathrm{~mm}$ focal distance)

\subsection{Acoustic detection}

Usually rotors of UAVs emit specific sound and therefore detection of UAVs is possible due to specific signature caused by fast rotating propellers (Hommes et al., 2016). For drone detection acoustic features are extracted and classified. It is possible to estimate the direction of the incoming sound and even elevation with a single acoustic antenna using classical beamforming algorithms, but obtaining distance measurement information is quite complicated in such approach. Therefore more complex systems with arrays of microphones (Busset et al., 2015) or even widely distributed acoustic on ground sensor complexes (Christnacher et al., 2016) are under development. This increases the capability of extracting a sound from a specific direction and more precise UAV localization by triangulation from different acoustic antenna positions. For portable acoustic systems usually a quite small drone detection range up to $250-300 \mathrm{~m}$ is reported (Hengy et al., 2017), therefore they are often coupled with other (video, LiDAR or RADAR) UAV detection solutions. Unfortunately, acoustic detection is completely ineffective against gliders or planes with temporary switched off engines.

\subsection{Passive RF detection}

Communication devices of UAVs usually emit relatively strong RF radiation in the desired frequency bands.
This radiation could serve as UAV presence indicator and could be received in kilometers of range. It is popular mean of detection of UAVs due to relatively low cost equipment requirements and operation at long range. Some drone detection scenarios by eavesdropping controller communication using low cost software defined radio (SDR) boards were tested in (P. Nguyen, Ravindranatha, A. Nguyen, Han, \& Vu, 2016). In addition to RF radiation, utilization of well-known wireless transmission protocols and techniques makes UAV presence detection possible using even simpler devices (Fu, Abeywickrama, Zhang, \& Yuen, 2018).

Unfortunately, UAV could fly with communication systems switched off. In this case this kind of detection becomes ineffective.

\subsection{RADAR detection}

Detection of small UAV using radar technology can be achieved using both active and passive radar systems. Passive radar system (e.g. Passive Bi-static Radar (PBR)) makes use of broadcast, communication or radio-navigation transmission signals to detect presence of objects. Transmitter and receiver are at separate locations and user have control of receivers only. The potential illumination signals, such as FM, DVB, GSM, GNSS or WIFI, could be used by PBR for UAV detection. Some experimental results of UAVs detection using DAB signal as illumination is reported in (Schüpbach, Patry, Maasdrop, Böniger, \& Wellig, 2017) and using DVB signal in (Liu et al., 2017).

The drawbacks of such radar are that waveforms it utilizes are not specifically designed for radar operation and consideration must be taken which transmission is best suited for specific targets. In addition to that, poor range and doppler resolution is observed of targets in short distance of radar elements, no matter the waveform used.

By principle of operation, active radars could be pulse or continuous wave (CW). A pulse radar transmits a very short, but high-power pulse and waits for the echo for the rest of its pulse repetition period, until next pulse is transmitted. Performance of such a radar is mainly influenced by the durations of transmitted pulse and echo receiving time window. Shorter the pulse duration, larger the bandwidth is which gives better range resolution. Pulse radars are mostly designed for long distances because of their high transmission power. Performance degrades at short distances due to shorter pulse duration need which leads to less energy dissipated for target illumination.

CW radar systems, on the other hand, continuously transmit an illumination signal and simultaneously continuously receive echo reflections scattered from objects. Moving object's speed and trajectory can be determined by observing its frequency shifts at receiver side due to Doppler effect. Ordinary CW systems are not able to perform range measurements without additional modulation, which encodes timing reference onto the transmit waveform. One of the most common modulation used is linear frequency modulation (FM) where particular value 
of frequency represents a particular time delay which corresponds to a particular range (Melvin \& Scheer, 2014).

Most of the current research involves FMCW radar usage for drone size UAV detection due to its low cost and power profile. Propositions using narrow beam FMCW radar to cover specific area are discussed in (Drozdowicz et al., 2016). As for pulse radar, field trials for small UAV detection using pulse coherent short range battlefield radar are reported in Ochodnický, Matoušek, Babjak, and Kurty (2017).

The performance of both active and passive radar detection heavily depends on Radar Cross Section (RCS) parameter which defines reflective strength of a target. Small UAVs, like quadcopter drones, usually are relatively small and not made from reflective materials which results that RCS values is in range of 0.001 to $5 \mathrm{~m}^{2}$. Any unwanted reflections from the scattering environment in the form of clutter will affect radar performance if the reflected power of the clutter is significantly above that of the radar system noise.

Main disadvantage of active radar system is that its performance highly depends on the price. Pulse magnetron radars provide wide blind zone and high power radiation. CW radar systems suffer from transmission signal leak in to receiver, unless specific design measures are taken to mitigate this affect.

\section{The approach of marine radar usage for drone detection}

Widest market of long-range radars is the market of marine radars. Development of the electronic industry over the past decades led to the mass production of low cost marine radars. Such radars, depending on their parameters are evaluated from 2 to 15 thousand of euro and have the weight mass of 7 to $25 \mathrm{~kg}$. Most of the low cost marine navigation radars have rotational scanning antennas. Depending on antenna width, antennas could be implemented as open array or closed dome antennas. By principle of operation marine radars are pulsed (power 1500-25000 W) magnetron radars, chirped pulsed (power 20-40 W) solid state radars and FMCW (power 0.2-0.4 W) solid state radars. Various types of radars provide various minimal distance to the target: $80 \mathrm{~m}$ and more for magnetron radar, $9 \mathrm{~m}$ for chirped pulse type radars, and $4.5 \mathrm{~m}$ for FMCW radars. Solid state marine radars are coherent. It provides possibility of Doppler shift selection of moving targets. Unfortunately, this option has not been implemented in FMCW radars of interest yet. In any case we consider solid state radar to be most perspective for drone detection.

Marine radars do not have clutter rejection function implemented which would give great detection advantage over the residential area. Thus, a possibility to connect radar to a PC could provide some further processing opportunity for minimal clutter rejection and additional target classification implementation. According to these issues, Simrad $4 \mathrm{G}^{\mathrm{TM}}$ and Simrad HALO ${ }^{\mathrm{TM}}$ radars were
Table 1. Technical specifications of Simrad $4 \mathrm{G}^{\mathrm{TM}}$ marine radar

\begin{tabular}{|l|c|}
\hline \multicolumn{1}{|c|}{ Specification type } & Value \\
\hline Operating frequency & X-band 9.3 to $9.4 \mathrm{GHz}$ \\
\hline Antenna width & Broadband FMCW \\
\hline Technology & $5.2+/-10 \%(-3 \mathrm{~dB}$ width $)$ \\
\hline Horizontal beam & $25+/-20 \%(-3 \mathrm{~dB}$ width $)$ \\
\hline Vertical beam & $0.4 / 0.6 / 0.8 \mathrm{~Hz}$ \\
\hline Scanning frequency & $22.17 \mathrm{dBm}(165 \mathrm{~mW})$ \\
\hline Output power (Antenna Port) & Up to $75 \mathrm{MHz}$ \\
\hline Sweep bandwidth & From 200 to $540 \mathrm{~Hz}$ \\
\hline Sweep repetition frequency & \\
\hline
\end{tabular}

chosen as possible candidates for investigation. Both of them can be used with commercial chart plotter device. Simrad $4 \mathrm{G}^{\mathrm{TM}}$ is also compatible with open source software (e.g. OpenCPN) which can be easily used as radar and chart plotter on PC. Due to lowest price and power, Simrad $4 G^{\mathrm{TM}}$ was chosen for a first experiments and Simrad $\mathrm{HALO}^{\mathrm{TM}}$ will be considered for further investigations. The brief specifications of Simrad $4 \mathrm{G}^{\mathrm{TM}}$ are provided in $\mathrm{Ta}-$ ble 1 . This radar is equipped with target separation control feature which, by changing its beam width, may provide additional benefits in small UAV detection scenarios.

\section{Measurement setup}

Simrad $4 G^{\mathrm{TM}}$ radar was mounted on the stand and placed on the roof of a SUV as presented in the (Figure 3). The total antenna height was approximately $3 \mathrm{~m}$. Simrad GO9 XSE chartplotter was used as radar display.

Two types of UAV were used as targets for radar detection measurement: DJI phantom 3 quadcopter and fixed wing UAV "Buzzard". DJI Phantom 3 quadcopter is a fully standard commercial product which represents the most common type of the UAVs used in these days.

The "Buzzard" UAV (Figures $4-5$ ) is a fixed wing $4 \mathrm{~kg}$ of weight UAV aircraft powered by electrical motor. The aircraft cruise speed is in range of $17 \mathrm{~m} / \mathrm{s}$, wingspan $1.6 \mathrm{~m}$ and the length $1.5 \mathrm{~m}$. The aircraft is manufactured from composite materials, mostly glass fiber, with minimal implementation of carbon fiber composite or any other radar reflective materials. Due to dynamic nature of the fixed wing UAV flight (the aircraft moves constantly) the detection of such vehicle is easier in comparison to the multi-

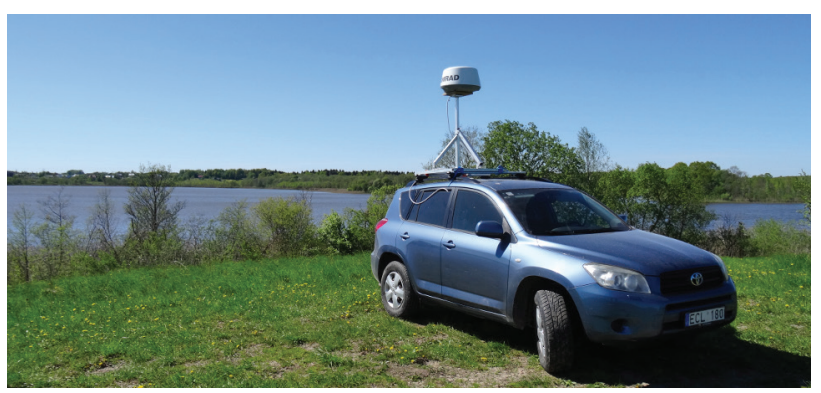

Figure 3. SIMRAD $4 \mathrm{G}^{\mathrm{TM}}$ radar mounted on SUV 
rotor type UAVs. During the experiment the aircraft was flying in $150 \mathrm{~m}$ radius circles over the certain spot which is clearly visible in Figure 7.

To ensure a clearer and unobstructed view during the experiment the flights were performed over the lake where minimal disturbing reflections were present.

\section{Results}

\subsection{Radar cross section measurements}

For preliminary estimation of radar's detection range, radar cross section (RCS) measurements of DJI Phantom drone were performed in fully anechoic chamber. Dimen-

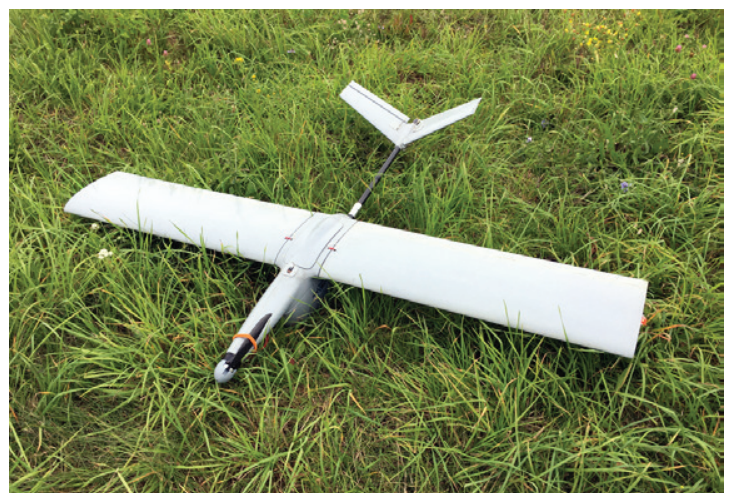

Figure 4. Fixed wing UAV "Buzzard"

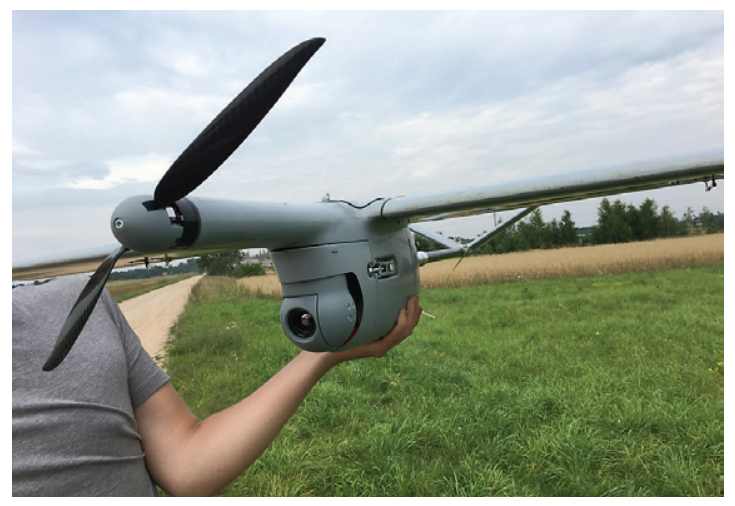

Figure 5. Fixed wing UAV "Buzzard”

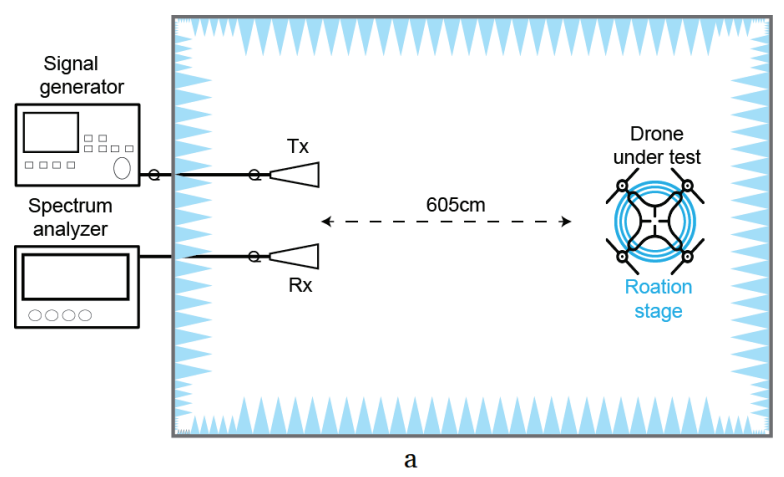

sions of the chamber are $8.4 \mathrm{~m} \times 4.6 \mathrm{~m} \times 3.7 \mathrm{~m}$. Measurement setup is shown in Figure 6a). The transmitting and receiving horn antennas were placed approximately $6 \mathrm{~m}$ from the drone under test. In order to reduce the cross talk to between both antennas the receiving antenna was placed $50 \mathrm{~cm}$ away from the transmitting one. To ensure high sensitivity, and repeatability of the measurements the drone was in the far field region of the antennas. The drone was placed on the rotational table and RCS's values on angle was measured rotating sample by $12.5^{\circ}$. A tunable microwave generator was used as a microwave source to generate $9.5 \mathrm{GHz}$ continuous wave signal. Approximately $P_{\mathrm{t}}=40 \mathrm{~mW}$ power signal was applied to the transmitting antenna. Signal reflected from the drone under test $P_{\mathrm{r}}$ was measured using spectrum analyzer, which was connected to the receiving antenna. Knowing the values of the transmitted and received signals it is possible to calculate radar cross section using formula:

$$
R C S=\frac{P_{r}(4 \pi)^{3} R^{4}}{P_{t} G_{t} G_{r} \lambda^{2}},
$$

where $R$ is the distance between antenna and drone under test, $\lambda$ - the wavelength of electromagnetic wave, $G_{\mathrm{t}}$ and $G_{\mathrm{r}}$ is gain of transmitting and receiving horn antennas.

Radar cross section measurements of DJI Phantom mounted with Hero 3 action camera is shown in Figure $6 \mathrm{~b})$. From the figure we can see that the Phantom has four RCS peaks at around $0^{\circ}, 180^{\circ}, 225^{\circ}$ and $250^{\circ}$. The maximum values at $0^{\circ}$ and $180^{\circ}$ can be anticipated due to the action camera placement. Maximum RCS value for this kind of drone is only around $0.09 \mathrm{~m}^{2}$. Also from the figure, we can observe that DJI Phantom has highly irregular RCS pattern, which could cause some problems when trying to find and track such a drone.

\subsection{Range measurements}

In order to reduce a clutter, measurements were performed over the lake. Map of the surrounding place is presented in the Figure 8. In the Figure 7 detection of fixed wing UAV is presented. UAV flies by circle trajectory around the island. Visualization of points of a track was switched

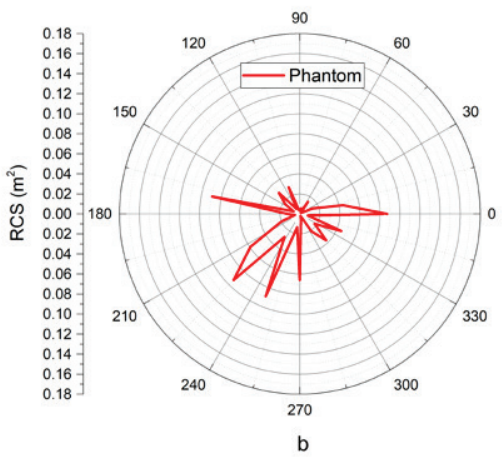

Figure 6. Radar cross section measurements: a - measurement setup in anechoic chamber; b - radar cross section measurement results of DJI Phantom drone 


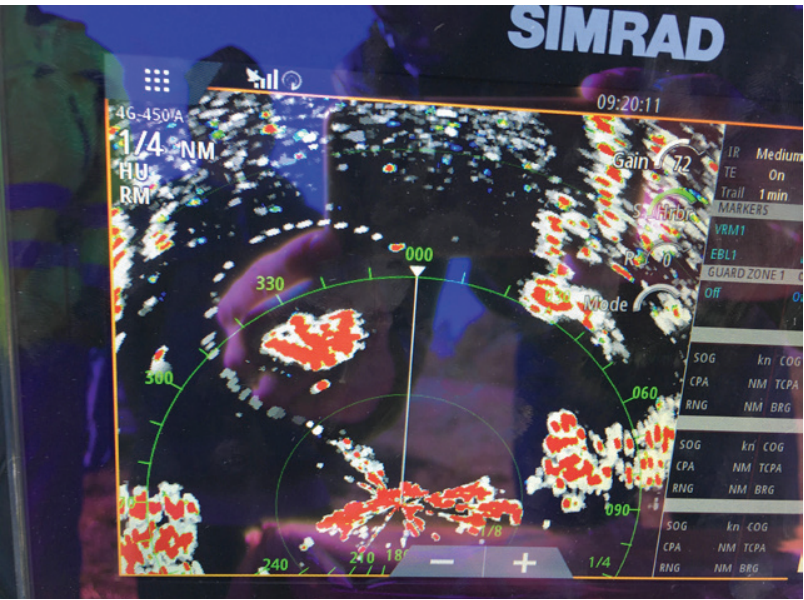

Figure 7. Fixed wing UAV detection over the lake using SIMRAD $4 \mathrm{G}^{\mathrm{TM}}$ radar

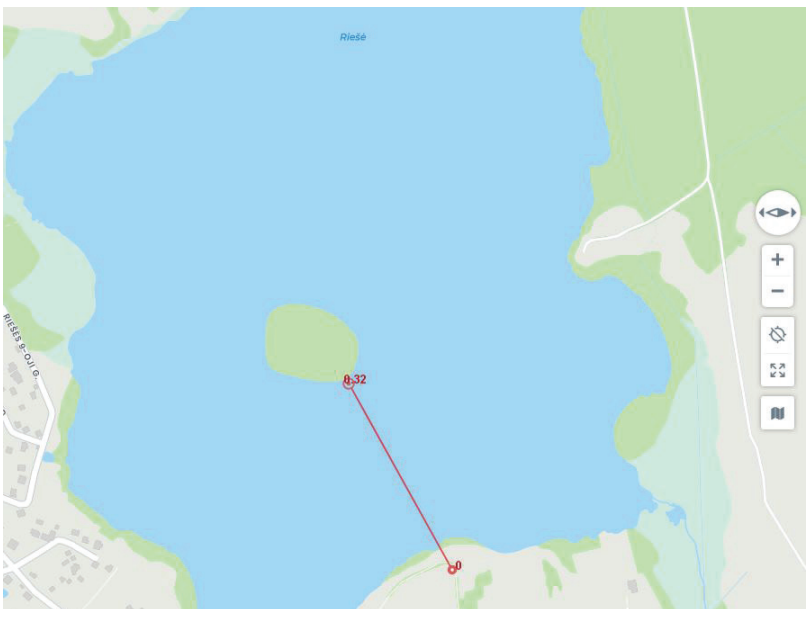

Figure 8 . The location of surrounding place where the tests were performed

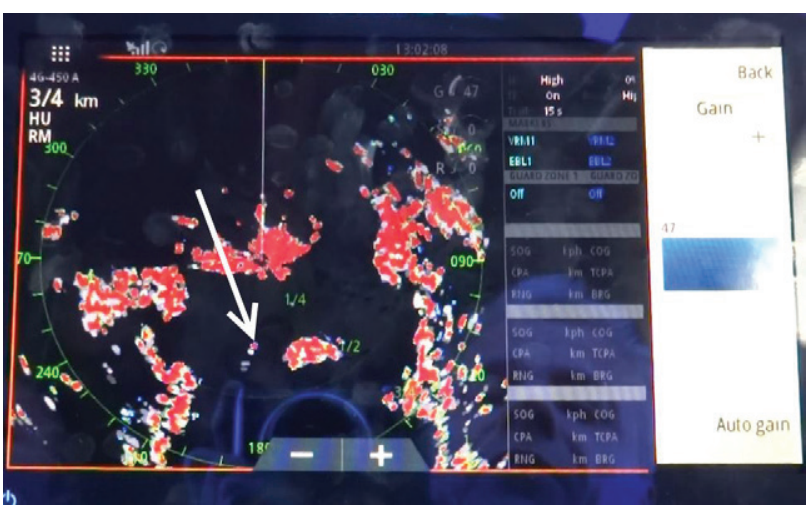

Figure 9. DJI Phantom detection over the lake using SIMRAD $4 G^{\text {TM }}$ radar

on. White dots are memorized tracking points. The range of reliable detection is approx. $600 \mathrm{~m}$.

Track of DJI Phantom quadcopter drone is presented in Figure 9. White arrow indicates current position of UAV. Detection range according to this figure is approximately $400 \mathrm{~m}$. Due to very irregular RCS of quadcopter (Figure 6b), track of flight flares. The drone is detectable in the range of $700 \mathrm{~m}$ when the drone is facing the radar in its maximum RCS value direction.

In both cases of measurement, appearance of a drone over the clutter makes a drone almost "invisible".

\section{Conclusions}

Detection of micro UAV by marine radar in the range of more than $500 \mathrm{~m}$ is possible. Detection is simpler in case of fixed wing UAV due to dynamic nature of its operations (it is easy to detect among the clutter), but detection of slow flying rotorcraft UAV is problematic. For maximum performance, radar should be arranged in the places with low clutter. Lack of clutter rejection option limits marine radar application for drone detection. Selection by Doppler effect and highlighting of moving targets would be useful feature for UAVs detection.

\section{Acknowledgements}

Research is funded by the European Regional Development Fund according to the supported activity "Targeted Research in Smart Specialization Areas" under Measure No. 01.2.2-LMT-K-718.
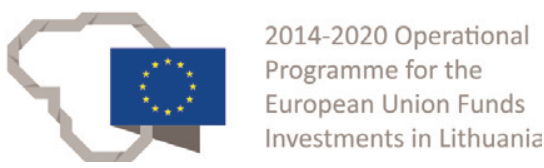

\section{References}

Busset, J., Perrodin, F., Wellig, P., Ott, B., Heutschi, K., Rühl, T., \& Nussbaumer, T. (2015). Detection and tracking of drones using advanced acoustic cameras. In Proceedings of the SPIE 9647, Unmanned/Unattended Sensors and Sensor Networks XI; and Advanced Free-Space Optical Communication Techniques and Applications, 96470F, Toulouse, France, 13 October 2015. https://doi.org/10.1117/12.2194309

Christnacher, F., Hengy, S., Laurenzis, M., Matwyschuk, A., Naz, P., Schertzer, S., \& Gwenael, S. (2016). Optical and acoustical UAV detection. In Proceedings of the SPIE 9988, ElectroOptical Remote Sensing X, 99880B, Edinburgh, United Kingdom, 21 October 2016. https://doi.org/10.1117/12.2240752

Drozdowicz, J., Wielgo, M., Samczynski, P., Kulpa, K., Krzonkalla, J., Mordzonek, M., Bryl, M., \& Jakielaszek, Z. (2016). $35 \mathrm{GHz}$ FMCW drone detection system. In 17th International Radar Symposium (IRS), Krakow, Poland, 10-12 May 2016. https://doi.org/10.1109/IRS.2016.7497351

Fu, H., Abeywickrama, S., Zhang, L., \& Yuen, C. (2018). Lowcomplexity portable passive drone surveillance via SDR-based signal processing. IEEE Communications Magazine, 56(4), 112-118. https://doi.org/10.1109/MCOM.2018.1700424

Hengy, S., Laurenzis, M., Schertzer, S., Hommes, A., Kloeppel, F., Shoykhetbrod, A., Geibig, T., Johannes, W., Rassy, O., \& Christnacher, F. (2017). Multimodal UAV detection: study of various intrusion scenarios. In Proceedings of the SPIE 10434, Electro-Optical Remote Sensing XI, 104340P. Warsaw, Poland, 5 October 2017. https://doi.org/10.1117/12.2278212 
Hommes, A., Shoykhetbrod, A., Noetel, D., Stanko, S., Laurenzis, M., Hengy, S., \& Christnacher, F. (2016). Detection of acoustic, electro-optical and RADAR signatures of small unmanned aerial vehicles. In Proceedings of the SPIE 9997, Target and Background Signatures II, 999701, Edinburgh, United Kingdom, 24 October 2016. https://doi. org/10.1117/12.2242180

Liu, Y., Wan, X., Tang, H., Yi, J., Cheng, Y., \& Zhang, X. (2017). Digital television based passive bistatic radar system for drone detection. In 2017 IEEE Radar Conference (RadarConf), Seattle, WA, USA, 08 May 2017.

https://doi.org/10.1109/RADAR.2017.7944443

Melvin, W. L., \& Scheer, J. A. (2014). Continuous wave radar. In W. L. Melvin \& J. A. Scheer, Principles of Modern Radar Applications (Vol. III, pp. 17-82). Edison, NJ, USA: SciTech Publishing.
Nguyen, P., Ravindranatha, M., Nguyen, A., Han, R., \& Vu, T. (2016). Investigating cost-effective RF-based detection of drones. In Proceedings of the 2nd Workshop on Micro Aerial Vehicle Networks, Systems, and Applications for Civilian Use (DroNet'16), Singapore, Singapore, 26 June 2016. (pp. 17-22). https://doi.org/10.1145/2935620.2935632

Ochodnický, J., Matoušek, Z., Babjak, M., \& Kurty, J. (2017). Drone detection by Ku-Band Battlefield Radar. In 2017 International Conference on Military Technologies (ICMT), Brno, Czech Republic, 31 May - 2 June 2017. https://doi.org/10.1109/MILTECHS.2017.7988830

Schüpbach, C., Patry, C., Maasdrop, F., Böniger, U., \& Wellig, P. (2017). Micro-UAV detection using DAB-based passive radar. In IEEE Radar Conference (RadarConf), Seattle, WA, USA, 8-12 June 2017.

https://doi.org/10.1109/RADAR.2017.7944357 